Témoigner Témoigner. Entre histoire et mémoire

Getuigen Revue pluridisciplinaire de la Fondation Auschwitz

$121 \mid 2015$

Violences radicales en scène

\title{
Entretien avec Hélios Azoulay
}

Daniel Weyssow

\section{OpenEdition}

Journals

Édition électronique

URL : https://journals.openedition.org/temoigner/3126

DOI : 10.4000/temoigner.3126

ISSN : 2506-6390

Éditeur :

Éditions du Centre d'études et de documentation Mémoire d'Auschwitz, Éditions Kimé

Édition imprimée

Date de publication : 1 octobre 2015

Pagination : 14-18

ISSN : 2031-4183

Référence électronique

Daniel Weyssow, «Entretien avec Hélios Azoulay », Témoigner. Entre histoire et mémoire [En ligne], 121 |

2015, mis en ligne le 01 octobre 2016, consulté le 04 février 2022. URL : http://

journals.openedition.org/temoigner/3126; DOI : https://doi.org/10.4000/temoigner.3126

Tous droits réservés 


\section{ENTRETIEN AVEC HELIOS AZOULAY}

MUSIQUE Compositeur, clarinettiste et écrivain, Hélios Azoulay a également été professeur d'histoire de la musique et d'histoire de l'art à l'université. Il a créé l'Ensemble de Musique Incidentale, voici 8 ans, avec la participation des musiciens Laurent Wagschal, Jonathan Bénichou, Teona Kharadze, Marielle Rubens, Giorgi Kharadze, Pablo Schatzman, Maud Lovett, Patrick Dussart, Baptiste Vay, Maja Bogdanovic, pour interpréter, notamment, des œuvres conçues dans les camps nazis.

$\rightarrow$ Entretien mené par Daniel Weyssow

eur premier $\mathrm{CD}$ enregistré, « ... même à Auschwitz (2014, premier opus de sa collection MUSIQUES D’OUTRE-MONDE) » est en soi, comme on l'aura compris, tout un programme. Un répertoire sobre et plutôt sérieux, qui sera prochainement suivi d'un second CD et d'un livre-disque conçu en collaboration avec Pierre-Emmanuel Dauzat, dont l'intitulé est « L'enfer aussi a son orchestre », attendu aux éditions La Librairie Vuibert. Il faut pourtant se rappeler que les premiers spectacles d'Hélios Azoulay, avant qu'il ne « sombre » dans la musique des camps, manifestaient un esprit bien particulier mêlant dadaïsme (sa composition Porte-bouteilles d'après l'œuvre de Marcel Duchamp), Pop Art (Boîte de soupe Campbell's d'après Andy Warhol), et musique expérimentale (John Cage, Steve Reich). Certains y ont même perçu l'un ou l'autre lien avec les situationnistes. Il a par ailleurs publié dans cet orbe deux essais éclairants intitulés Scandales! Scandales! Scandales! Histoires de chefs-d'œuvre que l'on siffle (Jean-Claude Lattès, 2008) et Tout est musique (La Librairie Vuibert, 2012). La présente interview d'Hélios Azoulay, réalisée

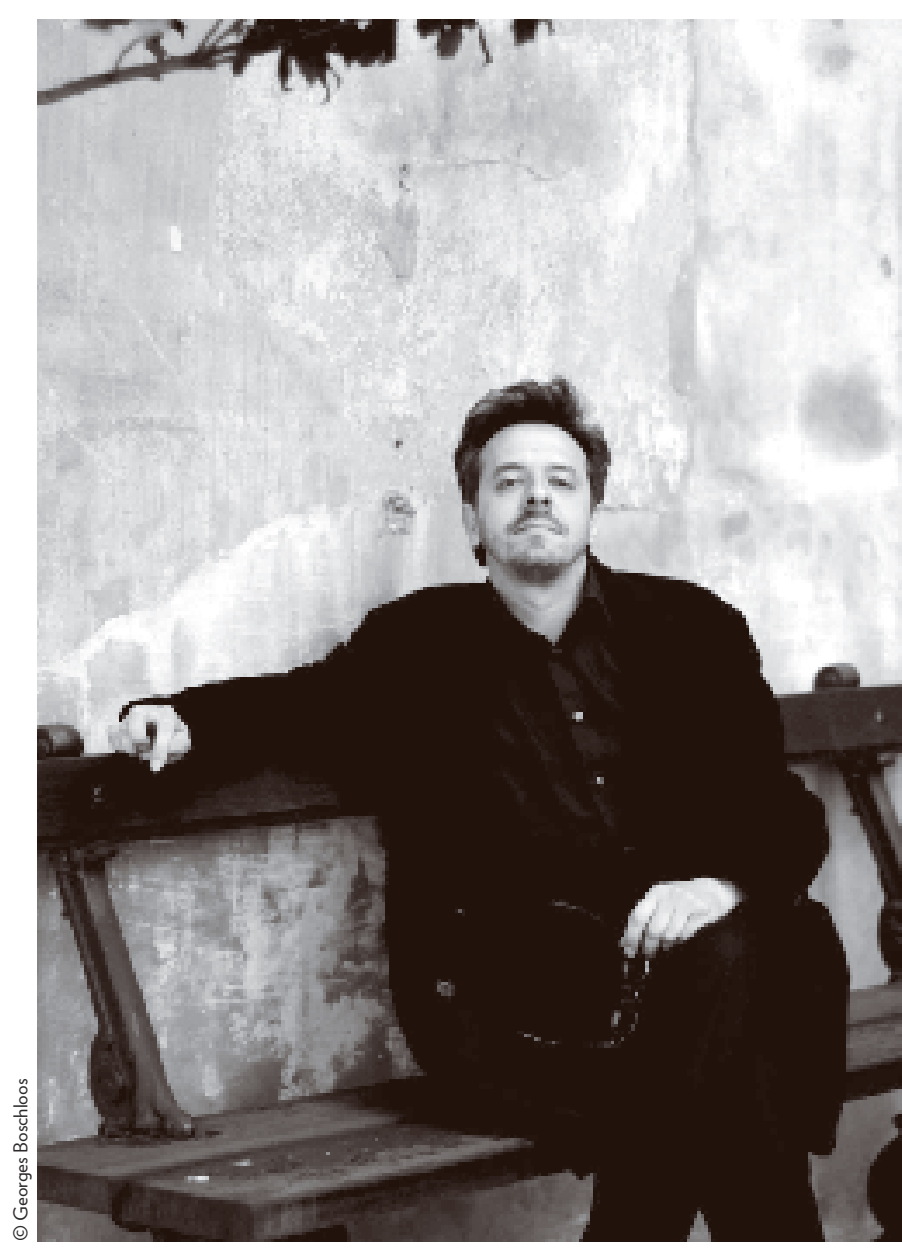

au lendemain d'un concert joué le 19 avril 2015 avec l'Ensemble de Musique Incidentale à l'Atelier Hastir à Bruxelles évoque sa démarche, ses choix musicaux ainsi que ses projets en cours. Moments choisis.

Pouvez-vous, brièvement, nous parler de votre démarche, de cet esprit de provocation, voire de révolte qui vous anime, mais aussi d'humour, qui traversent vos livres et vos spectacles?

Hélios Azoulay : Alors, déjà il faudrait bien évidemment s'entendre sur la question et la différence qu'il faut établir entre le désir de provocation et le fond de révolte. Car la provocation peut n'être (dans un sens lorsqu'elle est bassement stratégiquement publicitaire) 
qu'un moyen pour faire parler de soi. La révolte c'est un phénomène quand même beaucoup plus souterrain, beaucoup plus puissant, qui anime un certain nombre d'êtres dont je suis sans doute. Et ce qu'on appelle chez moi «provocation » n'est souvent qu'une réaction, une réplique. Il est vrai qu'un certain nombre de pièces, de concerts, ont effectivement fait scandale. Mais vous savez, le scandale est une manière très étonnante de remettre l'art dans le jeu de la vie, car le concert est parfois, comment dirais-je, une situation entendue de part et d'autre d'un plateau, dans lequel l'ennui se partage avec plus ou moins de passion. Dès lors que l’œuvre, comme ça m'est arrivé plusieurs fois, crée des remous dans la salle, fasse monter des gens sur scène, qu'on m'arrache la clarinette de la bouche, que les gens crient, etc., et bien vous êtes obligé de vous tenir de manière très héroïque et d'aller jusqu'au bout de ce que vous avez à dire. J'ai toujours été très étonné de faire scandale, parce que, vous savez, je pensais être écouté, et dès lors que vous n'êtes plus écouté, dès lors que vous êtes, comment dire, bousculés, et bien... c'est vraiment passionnant. Je crois que la chose la plus étonnante pour moi, à régler personnellement dans mon œuvre, c'est de faire rire. Parce que je me suis rendu compte en faisant des pièces sérieuses que les gens riaient. Ils trouvaient ça parfois ridicule, mais riaient parfois de bon cour. C'est quelque chose à apprivoiser, le fait de faire rire. Surtout musicalement. Voilà. Mais maintenant j'en suis très heureux, c'est une grande fierté même.

Pouvez-vous définir l'appellation d'Ensemble de Musique Incidentale que vous avez créé?

H.A. : Il est difficile de remonter le cours d'une sensibilité pour parvenir à la pulsion première d'un mot qui vous vient. Le terme Musique Incidentale par contre, je peux l'avouer, vient d'une pièce d'un compositeur Fluxus, donc du mouvement Fluxus, qui s'appelle George Brecht. Il a créé des tas de petites partitions sur des cartes de visite, qui ne sont pas plus grandes qu'une carte de visite, que nous pourrions échanger ensemble, dont une s'appelle Incidental Music. En anglais Incidental Music veut aussi dire «musique de scène », mais lui bouscule tout ça et il y a un deuxième mouvement qui est très beau dans cet Incidental Music : vous empilez les cubes les uns sur les autres à l'intérieur d'un grand piano, et ce mouvement s'achève dès lors que la petite pile de cubes tombe. Pour moi, la notion de Musique Incidentale est un processus que je mets en place. Je fais confiance au processus, qui m'amène finalement à découvrir ce qu'il y a à entendre.

Il vous est arrivé de manifester un esprit « dadaïste » dans vos spectacles. Est-il adaptable à notre époque? Êtes-vous arrivé à faire évoluer ce concept si toutefois il en était un?

H.A. : Pour moi ce n'est pas un concept, ce n'est même pas une période de l'histoire, c'est un état d'esprit. Marcel Duchamp, qui a été peut-être un des plus purs dadaïstes, un des plus détachés - et puisque la pureté est encore un autre problème -, reconnaissait l'esprit Dada chez Rabelais tout comme chez Alfred Jarry. À un moment, ce terme-là s'est cristallisé autour de la révolte et de l'engouement d'un certain nombre d'hommes, qui n'est pas sans lien avec la guerre 14-18, puisque né au cœur de ce moment-là à Zurich. Cet esprit, pour le coup, absolu de révolte, de provocation bien évidemment, c'est quelque chose qui existe encore. Donc il ne s'agit pas de savoir si on est $d a d a$, néo-dada ou post néo-dada, on peut être dadaïste sans que ça nous rattache au passé.

Voyez-vous un rapport entre votre intérêt pour ces musiques expérimentales et celles conçues dans les camps? Un parallélisme s'est-il dessiné, qui vous a orienté vers le chemin que vous avez emprunté?

H.A. : Il est vrai que c'est très étonnant. Cette musique m'a tout de suite claqué au visage. Après mon scandale à l'Opéra de Paris, qui est traité dans Scandales! Scandales! Scandales!, ce sabotage terrible du Jules César d'Haendel, qui a été rapporté par la presse, avoir cette réputation de saboteur, de post-dadaïste, néo-dadaïste, de tout ce que vous voulez d'extravagant, plonger là aurait pu paraître pour un certain nombre de gens une provocation de plus. Beaucoup d'artistes aujourd'hui, à des fins bassement marketing, se jettent sur la figure d'Hitler, du III ${ }^{\mathrm{e}}$ Reich, etc., ils se font ainsi de la publicité alors que pour moi, c'est le cadet de mes soucis. Ce que je sais, c'est qu'il y a quelque chose dans l'art de mon époque et dans la musique de mon époque qui m’apparaît être une très grande faille. Il y a une phrase dans les Lettres à un jeune poète de Reiner Maria Rilke, qui dit « une œuvre d'art est bonne qui surgit de la nécessité. » J'entends très peu d'œuvres musicales, aujourd'hui, qui surgissent de cette nécessité absolue intérieure. Notre époque musicale vit essentiellement sous perfusion de la culture. Il faudra bien faire un jour la différence entre la culture ou le culturel et l'art. Ce $\bullet \bullet$ 
•. que j’appelle le « subventionnisme », qui est absolument tentaculaire, nous cache les gens qui ont réellement des nécessités profondes de faire surgir d'eux une musique qui les dépasse. Parce que toutes nos œuvres sont des dépassements. Et dans les camps c'est exactement cela qui se pose, c'est-à-dire que cette sensibilité, celle que j'avais justement pour cette révolte, je la retrouve dans l'impulsion et la nécessité vitale et absolue de ces pauvres gens, de ces pauvres femmes, qui se sont mis à écrire, à chanter, dans un camp de concentration.

Allez-vous poursuivre vos concerts-spectacles empreints d'esprit dadaïste ou pensez-vous peu à peu être complètement absorbé par la musique des camps?

H.A. : C'est une question infiniment intéressante de plein de points de vue. Au départ c'est un sujet qui vous dépasse, et je crois qu'il n'en finira jamais de me dépasser. C'est même un sujet qui, si vous n'avez pas une certaine force morale, vous annihile. Ce qui veut dire que soit vous devenez le simple serviteur de cette mémoire - il y a des gens qui le font et c'est très bien -, soit vous essayez de vous en sortir. Quant à moi, quand j'examine l'histoire, avec le côté chaplinesque qu'on me reconnaît maintenant, je me rends compte que je n'ai rien à renier de ce que je suis profondément, c'est-à-dire en tout cas, je l'espère, un homme de joie, de rire, d'extravagance et d'outrance. De là vient l'énergie vitale qui me permet de rester en vie face aux berceuses d'Ilse Weber, face à toutes ces musiques terribles. Il suffit de regarder l'histoire. On n'aurait jamais fait confiance une seule seconde, au vu de son curriculum vitae, à Chaplin, s'il avait décidé de chercher des financeurs pour faire un Dictateur, n'est-ce pas? Et pourtant son film est une des preuves absolues que l'humour est un affront définitif et la possibilité de traiter avec «le sérieux du sourire», les sujets les plus insoutenables. Voilà une preuve de résistance absolue. Par le rire entre autres.

Comment concevez-vous les passerelles, les liens, entre musique, art et politique?

H.A. : Écoutez, c'est une question aussi qui nécessiterait qu'on y passe plusieurs heures (rires). Sans doute. Je crois, qu'on le veuille ou non, qu'une œuvre d'art, entendons-nous bien sur ce terme-là, est placée dans le jeu de l'histoire. Quand je dis placée, c'est comme sur un jeu d'échecs. Quand vous bougez un pion, vous modulez, vous changez définitivement le rapport de toutes les pièces présentes sur l'échiquier. Je crois qu'un artiste doit avoir conscience qu'il déplace des choses au sein d'une histoire qui elle aussi est mouvante. Quand il déplace quelque chose, il sait plus ou moins précisément les forces qu'il attire, les antagonismes qu'il crée, les puissances contradictoires qui vont s'affronter. Je pense qu'une œuvre, c'est presque un placement dans l'histoire. De l'histoire à la politique, il n’y a qu'un pas, facile à franchir.

Votre premier album, "... même à Auschwitz », comprend des morceaux principalement écrits à Terezín d'auteurs qui, la plupart, à l'exception d'Émile Goué et de Germaine Tillion, disparaîtront à Auschwitz : Karel Berman, Robert Dauber, Gidéon Klein, Hans Krása, Viktor Ullmann, Ilse Weber. Pourquoi avez-vous retenu ces compositeurs? Quels sont les critères qui ont présidé à vos choix? Leurs spécificités musicales, leur intensité, les lieux d'internement?

H.A.: Un disque, quand on prend le temps de l'écouter du début à la fin, c'est normalement quelque chose qui vous raconte si je puis dire une histoire. J'ai choisi ces pièces en fonction de leur succession qui raconte une histoire, mais au-delà de ça, quand on regarde l'œuvre d'Émile Goué, qui est un quatuor à cordes, quand on regarde Ilse Weber, qui sont des petites berceuses composées par une musicienne qui était une amatrice absolue, mélomane comme nous le sommes tous si je puis dire, quand on regarde Berman qui écrit une œuvre d'une férocité, d'une intensité pianistiquement inouïe - alors là il faut des doigts pour jouer Berman comme on dit vulgairement - , quand on regarde Klein qui était un immense compositeur, Ullmann également, qui était un élève de Schoenberg, l’idée de hiérarchie entre les musiques ou les genres s'effondre. Je vous citais Rilke tout à l'heure, une œuvre d'art est bonne qui surgit de la nécessité. C'est exactement ça. Ce que je subodorais instinctivement est éclatant. Il n'y a pas de hiérarchie entre un trio de Klein et une berceuse d’Ilse Weber. Toutes ces œuvres-là ont leur place pas tant dans l'histoire, que dans le mouvement vers l'art et la création. Et c'est ça que je trouve sublime. Toutes ces œuvres-là sont pour moi gorgées de la même densité.

Terezín, ville caserne située à une soixantaine de kilomètres de Prague, était un camp vitrine de la SS, un ghetto où les Juifs de Bohême-Moravie ont été rassemblés. La plupart ont été déportés à Auschwitz où ils ont été assassinés. Si le plus souvent les œuvres qui y ont été retrouvées ne laissent pas apparaître le fait qu'elles aient 

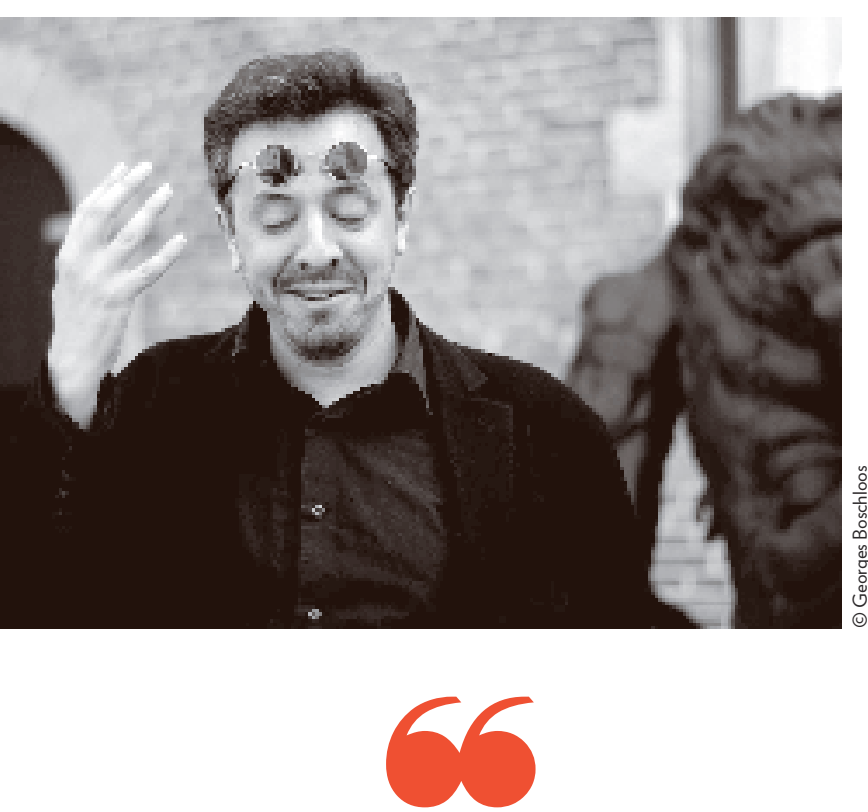

Le Dictateur est une des preuves absolues que l'humour est un affront définitif et la possibilité de traiter avec «le sérieux du sourire » les sujets les plus insoutenables

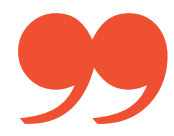

été conçues dans un camp de concentration, d'autres, au contraire, dénoncent le système concentrationnaire et le totalitarisme (Brundibár de Krása, Der Kaiser von Atlantis de Ullmann). Qu'en est-il des morceaux que vous interprétez?

H.A. : Je crois que l'art est dans certains cas une manière d'appuyer sur le réel, pour en extraire, si je puis dire, le pus. À l'inverse, vous avez cette sérénade de Dauber qui est d'une élégance, d'un envol et d'une aristocratie absolument sublime. C'est une pièce de salon que l'on pourrait imaginer faite par Kreisler [violoniste compositeur de pièces de salon, sans lien avec la déportation], pas du tout une pièce d'avant-garde, mais qu'importe, quelle élégance, quel courage pour trouver cette grâce dans la situation crasse, pourrie, infâme et infamante du camp de Terezin. C'est une manière de s'envoler du camp.

Vous avez découvert une composition inédite de Viktor Ullmann (auteur notamment de Der Kaiser von Atlantis [L'Empereur d'Atlantis] composée à Terezín) intitulée 30 mai 1431. Si le livret de cet opéra est achevé (vous le publierez en septembre au cœur de L'enfer aussi a son orchestre), seules deux pages de la partition ont été écrites. Pouvez-vous nous raconter l'histoire de cette découverte?

H.A. : La partition a été retrouvée par Francesco Lotoro, un italien qui a publié vingt-quatre disques sur la musique dans les camps. Un travail immense, une somme encyclopédique. C'est lui qui a trouvé ce 30 mai 1431. Je dois beaucoup au travail de Lotoro. Là, c'est donc de la seconde main, si je puis dire. Par contre je suis tombé sur un poème d'une beauté absolue et géniale en découvrant et en traduisant le livret d'Ullmann, qui est un grand compositeur. Il a aussi écrit des poèmes, que j'ai également traduits, ainsi que des aphorismes, que j'espère publier très vite. Ces deux pages de musique, puisqu'il n'y a eu que deux pages qu'il a eu le temps ou le désir ou que sais-je de composer, sont peut-être l'ouverture, ou le prélude de cet opéra, ou en tout cas le début du prélude, le fragment du prélude. Après vient le silence, qui me bouleverse. Je me suis dit qu'il fallait le terminer. Mais terminer non pas à la manière d'Ullmann parce que je ne vais pas prendre deux pages de musique pour en faire un opéra. Il faut l'entendre dire le texte, voilà. Ce que je désire à présent, ce à quoi je travaille, c'est en fait un travail de résurrection, mais avec mon langage. Je ne sais pas si c'est un travail d'historien, mais c'est peut-être aussi faire de l'histoire, au sens le plus classique du terme, que d'avoir envie de remettre ce texte, si je puis dire, dans le jeu de la vie.

Cet opéra évoque la mort de Jeanne d'Arc, brûlée vive sur un bûcher. La correspondance entre cette histoire et l'issue qui attendait Viktor Ullmann et les déportés de Terezín est évidente, mais elle est aussi complexe, comme l'expose Pierre-Emmanuel Dauzat, coauteur de votre livre cd, à paraître aux éditions La Librairie Vuibert, sur la musique des camps et leurs compositeurs, qui sera intitulé L'enfer aussi à son orchestre. Il décrit l'historique des apparitions et l'apparentement de l'épopée de Jeanne d'Arc qui, adaptée, se retrouve sous différentes formes $\ldots$.. 
•. dans l'histoire culturelle juive. Cet opéra de Viktor UIImann est une autre dénonciation claire et sans ambages d'Hitler et de ses sbires, du totalitarisme. Aurait-il été assassiné à Terezín en raison de la nature de sa nouvelle création?

H.A. : Der Kaiser von Atlantis n’a pu être représenté à Terezín. Ceux qui devaient créer l'œuvre ont été envoyés vers l'est, à Auschwitz. Cet opéra est une critique sublime, puissante, à peine voilée, du régime hitlérien. Comment connaître ou imaginer le destin de sa Jeanne d'Arc ?... Pourquoi a-t-il été déporté ? Vous savez, il est parti dans le même convoi, si ma mémoire est bonne, que Klein et Hans Krása. Les trois sont morts en même temps, le même jour. Un des rares qui s'en est sorti est le grand chef d'orchestre Karel Ančerl, qui a ensuite dirigé l'Opéra de Prague. Il y a eu une espèce d'effroyable hécatombe de tous ces musiciens de génie, le même jour, dans la même chambre à gaz. Alors qu'on ne vienne pas me seriner le lieu commun de la nation allemande mélomane...

Quelles sont les indications laissées par Viktor UIImann au sujet de cet opéra?

H.A. : Il a laissé deux pages sur ce qui pourrait être le prélude de l'œuvre. Elles mentionnent simplement une ligne mélodique de hautbois et trois trompettes. Il faut imaginer le reste, les cordes... Sinon il n'y a rien du tout. Mais c'est ça qui est très beau. Pour reprendre à peu près cette phrase de Sacha Guitry qui disait « après Mozart le silence qui reste est toujours de Mozart », moi je crois que le silence qui reste après Ullmann n'est pas d’Ullmann. C'est la tragédie de cette fin abrupte et c'est pourquoi il faut continuer.

Comment travaillez-vous les morceaux avec vos musiciens ? Est-ce que vous discutez ensemble de la manière dont ils devaient prendre forme? Apportent-ils chacun leur sensibilité, leurs connaissances de la musique des camps? Vous font-ils des suggestions?

H.A. : Alors, c'est moi qui leur donne les pièces. Je travaille beaucoup avec des gens qui ont déjà des carrières de chambristes ou de concertistes et qui jouent dans d'autres formations. Ce sont tous des musiciens accomplis. Il faut savoir laisser aux musiciens une part de liberté immense. Ce qui m'intéresse, c'est de retrouver le plus possible, chez Berman, une fureur, chez Ilse Weber, une grâce. Marielle Rubens, qui chante, n'a pas du tout une voix d'opéra. C'était la limite du disque, édité chez Deutsche Grammophon, de la cantatrice
Anne Sophie von Otter dont l'interprétation de pièces créées dans les camps m'avait malgré tout bouleversé. Je crois que c'est un répertoire qui ne supporte pas la boursouflure. C'est un répertoire qui ne supporte pas qu'on vienne y coller l'histoire de la musique en somme. C'est pour ça que cette musique est à la fois, dans l'histoire de l'art comme dans l'histoire de l'humanité, une incohérence absolue. Et c'est peut-être là-dedans que se retrouvent les germes de dada dont on parlait tout à l'heure. Il y a quelque chose de l'ordre de la fracture, de l’incohérence absolue. La folie définitive.

Pour nous résumer et conclure, deux petites questions encore. $Y$ a-t-il un message particulier que vous souhaiteriez insuffler au travers des morceaux que vous interprétez?

H.A. : Alors le terme de «message » c'est un peu comme le terme « pédagogie », il me dérange, différemment, mais me dérange aussi. Les témoins directs vont disparaître. J'en ai vu disparaître qui étaient des gens que j'adorais. C'est une tragédie inéluctable. C'est celle de la vie et du temps qui passe. Maintenant les hommages se multiplient. Parfois on y va aussi par soustraction, il y en a de moins en moins aussi. Mais je veux dire qu'on ne peut pas laisser à l'hommage, au silence - l'hommage à l'air grave qu'on prend dans ces circonstances -, enterrer la vitalité. On ne peut pas laisser à l'hommage le soin d'enterrer un hommage, qui enterre un autre hommage, qui va enterrer un nouvel hommage. Il nous faut maintenant de nouveaux moyens de paroles. Pas tant pour rappeler une histoire que pour susciter la foi profonde, que nous possédons tous en nous, le pouvoir de résister.

Quel devrait être, quel est, selon vous, la place, le rôle du créateur dans notre monde, aujourd'hui ?

H.A.: Écoutez, le créateur authentique est indispensable, pour changer le monde, pour donner. Je crois aux poètes, aux poètes authentiques. Je crois qu'un jour ou l'autre, on remettra la main sur ces hommes qui sont capables de mettre entre deux imbéciles une phrase, au milieu, pour les réconcilier (rires). 